\title{
First-principles calculation of optical absorption spectra in conjugated polymers: Role of electron-hole interaction
}

\author{
Michael Rohlfing \\ Institut für Theoretische Physik II - Festkörperphysik, Universität Münster, Wilhelm-Klemm-Straße 10, 48149 Münster, \\ Germany \\ M. L. Tiago and Steven G. Louie \\ Department of Physics, University of California at Berkeley, Berkeley, CA 94720, USA, and Materials Sciences Division, \\ Lawrence Berkeley National Laboratory, Berkeley, CA 94720, USA.
}

(September 20, 2000)

\begin{abstract}
Experimental and theoretical studies have shown that excitonic effects play an important role in the optical properties of conjugated polymers. The optical absorption spectrum of transpolyacetylene, for example, can be understood as completely dominated by the formation of exciton bound states. We review a recently developed first-principles method for computing the excitonic effects and optical spectrum, with no adjustable parameters. This theory is used to study the absorption spectrum of two conjugated polymers: trans-polyacetylene and poly-phenylene-vinylene (PPV).
\end{abstract}

\section{INTRODUCTION}

During the last two decades, conjugated polymers have become an important field of study due to the very special properties of these materials: besides having the desired mechanical properties of polymers, they can be made good electric conductors when doped. Semiconducting polymers are also efficient for use in optical devices such as lightemitting diodes [1]. Our basic understanding of these materials is however far behind that of the conventional semiconductors. An accurate theoretical study of conjugated polymers would contribute to a better understanding of their properties. With advanced $a b$ initio methods, one should be able to predict new properties and phenomena associated with these materials. In fact, ab initio excited-state calculations with the inclusion of quasiparticle effects $[2,3]$ have been able to predict from first principles the one-particle excitation spectrum of many semiconductors, with good agreement with experiment. A recently developed theoretical approach [4-6] based on solving the Bethe-Salpeter equation (BSE) of the two-particle Green's function [7] also has been successfully applied to the optical spectra of many semiconductor systems, as well as polymers [8], with the interaction between the excited electron and hole explicitly treated. In particular, the energy and wavefunction of the coupled electron-hole states are obtained [4], and the imaginary part of the dielectric function, related to the optical absorption of the material, is calculated from first principles. In this article, we review the theoretical framework of this approach and discuss some of the results obtained for polymers. We discuss the theoretical methods in section II. In section III, the calculated results for two polymers, trans-polyacetylene and PPV, are presented together with some experimental data. The first system is studied both as a single chain and in a crystalline environment. We summarize with some final comments and conclusion in section IV.

\section{THEORETICAL METHODS}

The Hohenberg-Kohn-Sham density functional theory (DFT) has been shown to give a good description of the ground-state properties for a variety of systems, including polymers [8,9]. It is a self-consistent field theory that describes the ground-state properties, such as the charge density and total energy of the system, in terms of a set of $N$ one-electron orbitals $\varphi_{j}(\mathbf{r})(N=$ number of electrons) that satisfy the Kohn-Sham equations:

$$
\left[-\frac{\hbar^{2} \nabla^{2}}{2 m}+V_{e x t}(\mathbf{r})+\int \frac{e^{2} \rho\left(\mathbf{r}^{\prime}\right)}{\left|\mathbf{r}-\mathbf{r}^{\prime}\right|} \mathrm{d}^{3} \mathbf{r}^{\prime}+V_{x c}(\mathbf{r})\right] \varphi_{n, \mathbf{k}}(\mathbf{r})=\varepsilon_{n, \mathbf{k}} \varphi_{n, \mathbf{k}}(\mathbf{r})
$$

Here, $V_{e x t}$ is the potential generated by the ions. Exchange and correlation effects are included in the exchangecorrelation potential $V_{x c}$. In practice, this potential is unknown, and it is modeled within the local density approximation (LDA) or generalized gradient approximation (GGA), the latter being more appropriate for systems with large inhomogeneity in charge density. In the calculations, we solve Eq. (1) self-consistently, with the orbitals expanded 
either in a set of plane waves or Gaussian-orbital basis functions [10]. The polymer is treated as infinitely long. The theory is also able to predict the equilibrium, ground state geometry by minimizing the total energy with respect to the position of each atom. However, the Kohn-Sham eigenvalues cannot be directly interpreted as the energy of the quasiparticle excitations of the interacting electron system [2].

To obtain the single-particle excitations, one needs a quasiparticle theory that takes into account the fact that the simplest excitations, quasi-electrons and quasi-holes, have wavefunctions and excitation energies different from their non-interacting counterparts. The quasiparticle properties can be found by solving a Dyson's equation from the single-particle Green's function,

$$
\left[-\frac{\hbar^{2} \nabla^{2}}{2 m}+V_{e x t}(\mathbf{r})+\int \frac{e^{2} \rho\left(\mathbf{r}^{\prime}\right)}{\left|\mathbf{r}-\mathbf{r}^{\prime}\right|} \mathrm{d}^{3} \mathbf{r}^{\prime}\right] \varphi_{n, \mathbf{k}}^{q p}(\mathbf{r})+\int \mathrm{d}^{3} \mathbf{r}^{\prime} \Sigma\left(\mathbf{r}, \mathbf{r}^{\prime} ; E_{n, \mathbf{k}}^{q p}\right) \varphi_{n, \mathbf{k}}\left(\mathbf{r}^{\prime}\right)=E_{n, \mathbf{k}}^{q p} \varphi_{n, \mathbf{k}}^{q p}(\mathbf{r})
$$

where $\Sigma$ is the self-energy operator. In the GW approximation [2], this operator is written as a product of the Green's function, $G\left(\mathbf{r}, \mathbf{r}^{\prime}\right)$, and the screened Coulomb interaction, $W$, between the electrons. As discussed in Ref. [2], $W$ may be evaluated from first principles using an RPA dielectric function.

In practice, Eq. (2) is evaluated in the basis given by the Kohn-Sham orbitals. Generally, the quasiparticle wavefunctions and Kohn-Sham orbitals are quite similar $[2,11]$. In this case, the QP energy of a state $\{n, \mathbf{k}\}$ can be written to first order as

$$
E_{n, \mathbf{k}}^{q p}=\varepsilon_{n, \mathbf{k}}+\left\langle\varphi_{n, \mathbf{k}}\left|\Sigma\left(\mathbf{r}, \mathbf{r}^{\prime} ; E_{n, \mathbf{k}}^{q p}\right)-V_{x c}(\mathbf{r})\right| \varphi_{n, \mathbf{k}}\right\rangle
$$

In the Green's function formalism, once the QP wavefunctions and excitation energies are available, we may consider other types of excitations, such as the coupled electron-hole excitation in optical processes. Optical properties require a two-particle description that takes into account the interaction between the quasi-electron and hole involved in the absorption/emission of light. The Bethe-Salpeter equation of the two-particle Green's function provide us with a highly accurate description of optical properties in systems where electron-hole interactions are important. The details of the theoretical method are discussed in [4-7]. In our approach [4], the energy and wavefunction of such excited many-electron states are explicitly calculated from the quasiparticle states obtained from the GW calculation.

The coupled electron-hole excited states are taken to be of the form $|S\rangle=\sum_{c v \mathbf{k}} A_{c v \mathbf{k}} a_{c \mathbf{k}}^{\dagger} a_{v \mathbf{k}}|G\rangle$, where $|G\rangle$ is the ground state (that is, with no excited quasiparticles), and $A_{c v \mathbf{k}}$ is a probability amplitude of having an excited electron at state $\{c, \mathbf{k}\}$ and a hole at $\{v, \mathbf{k}\}$. The possibility of multiple-pair processes is ignored in the regime of linear optics, as is also the near zero momentum transfer between the incident photon and the electron-hole pair. The coefficients $A_{c v \mathbf{k}}$ satisfy the secular equation $[4,7]$

$$
\left(E_{c, \mathbf{k}}^{q p}-E_{v, \mathbf{k}}^{q p}\right) A_{c v \mathbf{k}}+\sum_{c v \mathbf{k}, c^{\prime} v^{\prime} \mathbf{k}^{\prime}} \mathcal{K}_{c v \mathbf{k}, c^{\prime} v^{\prime} \mathbf{k}^{\prime}} A_{c^{\prime} v^{\prime} \mathbf{k}^{\prime}}=\Omega A_{c v \mathbf{k}}
$$

known as the Bethe-Salpeter equation. $\Omega$ is the excitation energy of the state $|S\rangle$, and $\mathcal{K}$ is the interaction kernel. The interaction $\mathcal{K}$ has two contributions: an attractive direct interaction involving the screened Coulomb potential between electron and hole, $\mathcal{K}_{c v \mathbf{k}, c^{\prime} v^{\prime} \mathbf{k}^{\prime}}$, and a repulsive exchange interaction involving the bare Coulomb potential, $\mathcal{K}_{c v \mathbf{k}, c^{\prime} v^{\prime} \mathbf{k}^{\prime}}$. The exchange interaction is allowed in spin singlet excitonic states, where the full interaction kernel is $\mathcal{K}=\mathcal{K}^{d}+2 \mathcal{K}^{x}$ (implicit indices), but not in spin triplet excitonic states, hence $\mathcal{K}=\mathcal{K}^{d}$. In the static screening limit, the expressions for the exchange and direct terms in $\mathcal{K}$ are given below.

$$
\begin{gathered}
\mathcal{K}_{c v \mathbf{k}, c^{\prime} v^{\prime} \mathbf{k}^{\prime}}^{x}=\int \mathrm{d}^{3} \mathbf{r} \mathrm{d}^{3} \mathbf{r}^{\prime} \varphi_{v, \mathbf{k}}\left(\mathbf{r}^{\prime}\right) \varphi_{c, \mathbf{k}}^{*}\left(\mathbf{r}^{\prime}\right) \frac{e^{2}}{\left|\mathbf{r}-\mathbf{r}^{\prime}\right|} \varphi_{v^{\prime}, \mathbf{k}}^{*}(\mathbf{r}) \varphi_{c^{\prime}, \mathbf{k}}(\mathbf{r}) \\
\mathcal{K}_{c v \mathbf{k}, c^{\prime} v^{\prime} \mathbf{k}^{\prime}}^{d}=-\int \mathrm{d}^{3} \mathbf{r}^{3} \mathbf{r}^{\prime} \varphi_{v, \mathbf{k}}\left(\mathbf{r}^{\prime}\right) \varphi_{v^{\prime}, \mathbf{k}}^{*}\left(\mathbf{r}^{\prime}\right) W\left(\mathbf{r}, \mathbf{r}^{\prime}\right) \varphi_{c, \mathbf{k}}^{*}(\mathbf{r}) \varphi_{c^{\prime}, \mathbf{k}}(\mathbf{r})
\end{gathered}
$$

An important quantity that is affected by electron-hole interactions is the macroscopic dielectric function. In particular, the imaginary part of the dielectric function can be written as 


$$
\epsilon_{2}(\omega)=\frac{4 \pi^{2} e^{2}}{\omega^{2}} \frac{1}{V_{c}} \sum_{S}|\langle G|\lambda \cdot \mathbf{v}| S\rangle|^{2} \delta\left(\Omega_{S}-\hbar \omega\right)
$$

where the summation goes over all transitions, from ground state $|G\rangle$ to excited state $|S\rangle$. $\Omega_{S}$ is the corresponding excitation energy, $\mathbf{v}$ is the velocity operator, and $\lambda$ is the polarization vector. The volume of the crystal, $V_{c}$, ensures that the summation over states gives a finite quantity. If the excited state is assumed to have non-interacting electron and hole, $|S\rangle=a_{c \mathbf{k}}^{\dagger} a_{v \mathbf{k}}|G\rangle$, then this function reduces to

$$
\epsilon_{2}^{(0)}(\omega)=\frac{4 \pi^{2} e^{2}}{\omega^{2}} \frac{1}{V_{c}} \sum_{c v \mathbf{k}}|\langle v \mathbf{k}|\lambda \cdot \mathbf{v}| c \mathbf{k}\rangle|^{2} \delta\left(E_{c, \mathbf{k}}^{q p}-E_{v, \mathbf{k}}^{q p}-\hbar \omega\right)
$$

Electron-hole interactions lead to a coupling of pair configurations in the excited state, that takes the assumed form $|S\rangle=\sum_{c v \mathbf{k}} A_{c v \mathbf{k}} a_{c \mathbf{k}}^{\dagger} a_{v \mathbf{k}}|G\rangle$. In this case, the dielectric function is

$$
\epsilon_{2}(\omega)=\frac{4 \pi^{2} e^{2}}{\omega^{2}} \frac{1}{V_{c}} \sum_{S}\left|\sum_{c v \mathbf{k}} A_{c v \mathbf{k}}^{S}\langle v \mathbf{k}|\lambda \cdot \mathbf{v}| c \mathbf{k}\rangle\right|^{2} \delta\left(\Omega_{S}-\hbar \omega\right)
$$

Another important quantity obtainable from our calculation is the electron-hole wavefunction: $\chi_{s}\left(\mathbf{r}_{h}, \mathbf{r}_{e}\right)=$ $\sum_{c v \mathbf{k}} A_{c v \mathbf{k}}^{S} \varphi_{v \mathbf{k}}^{*}\left(\mathbf{r}_{h}\right) \varphi_{c \mathbf{k}}\left(\mathbf{r}_{e}\right)$, which describes the correlation between the excited electron and hole.

\section{RESULTS}

The above approach was first applied to an infinite, isolated chain of trans-polyacetylene [8]. This is the simplest conjugated polymer [1]. Since the structure of this polymer is well known from NMR and X-ray measurements [12,13], we use these data for the position of the atoms in our calculation. The fact that real samples contain chains of finite length does not conflict much with the infinitely long chain assumption since the size of typical excitonic states is shorter than the typical chain length [1]. In the calculation, all atoms are kept stationary. An important structural parameter is the dimerization distortion, i.e. the difference in length between the carbon single bonds and double bonds, which we take from experiment.

The quasiparticle band structure for trans-polyacetylene, calculated with a Gaussian basis set are presented in Figure 1a. The highest occupied band and lowest unoccupied band are formed from delocalized $\pi$ states, with the expected symmetry properties. These extended bands play an important role in the conductivity of doped polyacetylene [1]. A direct LDA energy gap of $0.74 \mathrm{eV}$ is found at the $X$ point, on the border of the Brillouin zone. Similar results for the band structure and energy gap are obtained in a DFT-GGA calculation, using a plane wave basis set. The GGAcomputed energy gap is $0.78 \mathrm{eV}$, in good agreement with the previous result within the precision of the theory. The width of the $\pi$ bands and the lower occupied bands also agree in both calculations. The QP band structure (spectrum of one-particle excitations) is qualitatively similar to the one at the DFT level. The most relevant difference is an almost rigid shift of energy bands so as to increase the energy gap, that is now $2.1 \mathrm{eV}$.

Using the QP results, we solved the Bethe-Salpeter equation and evaluated the optical absorption spectrum of trans-polyacetylene. Figure 2 shows the calculated absorption strength, i.e. the imaginary part of the dielectric function. The polarization vector is taken along the chain direction. The dashed line shows the dielectric function without electron-hole interaction, given by Eq. (7). The sharp peak at $E_{g}=2.1 \mathrm{eV}$ corresponds to vertical $\pi \rightarrow \pi^{*}$ transitions, and it diverges as $\left(\hbar \omega-E_{g}\right)^{-1 / 2}$. This behavior comes directly from a one-dimensional joint density of states, with onset at the gap energy. Transitions involving other bands occur at higher energies.

With electron-hole interactions, the absorption spectrum changes considerably. The peak at $E_{g}$ is suppressed by a destructive coherent coupling of the optical matrix elements of the electron-hole pair configurations in the formation of the excitations (see Eq. 8). Constructive coherent coupling of these matrix elements for a bound exciton give rise to a sharp peak positioned at $1.7 \mathrm{eV}$. This corresponds to formation of an optically active exciton with the same energy, in agreement with spectroscopic measurements that showed a maximum in $\epsilon_{2}(\omega)$ exactly at $1.7 \mathrm{eV}$ [14]. Exciton-phonon coupling and/or static lattice distortion are expected to be responsible for a broadening of the exciton peak found in the experiment. A second exciton, with excitation energy $1.8 \mathrm{eV}$, is also found in the calculation but it is optically inactive. The absorption spectrum with polarization perpendicular to the chain direction, but still in 
the polymer plane, is found to be 3 orders of magnitude weaker than the case with polarization along the chain. This is also in agreement with experimental data $[8,14]$. No significant optical absorption was observed with polarization perpendicular to the polymer plane. This anisotropy is explained by the reduced polarizability of $\pi$ electrons at directions perpendicular to the chain. At high energy (above $8 \mathrm{eV}$ ) some absorption is observed with polarization perpendicular to the chain, due to transitions other than $\pi \rightarrow \pi^{*}$.

In order to study interchain interactions in trans-polyacetylene, we have considered the crystalline phase of this polymer. The geometrical conformation is taken from crystallographic data [13], and the chains are again considered infinitely long. Trans-polyacetylene crystallizes in a monoclinic phase, with 2 monomers (in different chains) per unit cell. The effect of interchain interactions is visible in the band structure (obtained from DFT-GGA calculation) in Fig. 3. The energy splitting, particularly at the points $\Gamma$ and $A$ in the first Brillouin zone are directly related to overlapping between atomic wavefunctions sitting on neighboring chains. The small splitting indicates weak overlap. Indeed, the distance between neighbor chains is rather large: the smallest distance between carbon atoms in different chains is about $4 \AA$. This is to be compared with the length of the carbon single bonds and double bonds $(1.45 \AA$ and $1.36 \AA$ respectively). Another signature of weak overlap can be found in the reduced band dispersion along the paths $D B A E D B$ and $Y C X \Gamma$, both perpendicular to the chain direction. Within DFT-LDA, there is a direct energy gap of $0.47 \mathrm{eV}$ at the $A$ point. These findings are in agreement with earlier theoretical calculations, using LDA [9,15] (see Table I).

The inclusion of quasiparticle self-energy effects increases considerably the energy gap, to $2.1 \mathrm{eV}$. It is still direct and located at the $A$ point. This value is about $0.2 \mathrm{eV}$ larger than what was reported by Ethridge $e t$ al., who used a Hartree-Fock model with quasiparticle effects [15]. It is also larger than the experimental estimate [14], based on the onset of the absorption coefficient as function of energy. Since excitonic effects have been demonstrated to be so strong in the single chain case, the inclusion of electron-hole interaction effects in the crystal case is expected to yield an optical gap close to the value of $1.7 \mathrm{eV}$ found above.

The next system studied with the presented BSE approach was poly-phenylene-vinylene (PPV) [8]. It has 14 atoms in the unit cell, and was also studied as a single polymeric chain. The quasiparticle band structure is depicted in Figure 1b. This polymer has a direct quasiparticle energy gap of $3.3 \mathrm{eV}$, at the $X$ point. The highest valence band and lowest conduction band consist of extended states with wavefunctions having $\pi$ orbitals on all the carbon atoms in the chain, thus resulting in dispersive bands. On the other hand, the next bands (denoted $\pi_{2}$ and $\pi_{2}^{*}$ in Figure 1b) are nearly dispersionless, with states localized on the phenylene rings. The distribution of atomic orbitals of states in each one of these bands is as deduced from the electronic structure of PPV in previous studies [18].

The absorption strength of PPV is also strongly modified by excitonic effects (see the solid curve in Fig. 4). The dashed line in Fig. 4 shows the absorption strength calculated without electron-hole interactions. The lowest energy peak corresponds to interband transitions from highest occupied band to lowest unoccupied band. Higher energy structures at near $5.5 \mathrm{eV}$ are related to transitions $\pi_{1} \rightarrow \pi_{2}^{*}$ and $\pi_{2} \rightarrow \pi_{1}^{*}$, and the peak at $8 \mathrm{eV}$ corresponds to $\pi_{2} \rightarrow \pi_{2}^{*}$ transitions. Including electron-hole interaction, these peaks are suppressed, and the spectrum is dominated by several new sharp peaks associated with exciton formation. The first one is located at $2.4 \mathrm{eV}$, in good agreement with experimental results that show a sharp peak at $2.5 \mathrm{eV}$. This exciton state thus has a binding energy of $0.9 \mathrm{eV}$ with respect to the quasiparticle band gap. Other features include two sharp peaks at $4.0 \mathrm{eV}$ and $4.9 \mathrm{eV}$ and several structures in the range of $5.8-6.9 \mathrm{eV}$. The calculated results thus explain well the observed experimental features at $3.7 \mathrm{eV}, 4.8 \mathrm{eV}$ and $6.0 \mathrm{eV}[8,16]$. Since these peaks occur above the energy gap, these exciton states actually interact with the free electron-hole continuum and should be interpreted as resonant states rather than bound exciton states. The profiles of the calculated exciton states at $2.4 \mathrm{eV}$ and $4.0 \mathrm{eV}$ are illustrated in Fig. 5, showing that the state at $2.4 \mathrm{eV}$ is a bona fide bound state but the one at $4.0 \mathrm{eV}$ is a quasi-bound or resonant state. The present results are qualitatively similar to results obtained in several previous model Hamiltonian studies [17-19].

\section{CONCLUSION}

In summary, we have discussed the optical absorption spectrum and excitonic wavefunctions of two conjugated polymers. The calculated results are from a first-principles approach based on solving the single-particle and twoparticle Green's function of the interacting electron system, with the full Hamiltonian. Good agreement has been achieved with the measured spectra as well as with previous theoretical results that involved model Hamiltonians. 
The optical spectrum, both for trans-polyacetylene and poly-phenylene-vinylene, is dominated by peaks associated with bound and resonant excitonic states. In particular, the optical gap is found to be significantly smaller than the quasiparticle gap, showing a large excitonic binding energy with respect to the free quasiparticle energies. However, if it is defined with respect to the polaronic gap (where lattice relaxation is included), the binding energy would be smaller.

\section{ACKNOWLEDGMENTS}

This work was supported by the Deustche Forschungsgemeinschaft (Bonn, Germany) under Grant No. Ro-1318/1-1, by National Science Foundation Grant No. DMR-9520554, and by the Director, Office of Energy Research, Office of Basic Energy Sciences, Materials Sciences Division of the U.S. Department of Energy under Contract No. DE-AC0376SF00098. Computational resources have been provided by the National Energy Research Scientific Computing Center (NERSC). 
TABLE I. Calculated energy gap of trans-Polyacetylene (in eV)

\begin{tabular}{lll}
\hline \hline & single chain & crystal (P2 $/ n)$ \\
\hline Kohn-Sham gap (LDA) & 0.74 & - \\
Kohn-Sham gap (GGA) & 0.78 & 0.47 \\
QP gap (GW) & 2.1 & 2.1 \\
LDA, ref. [9] & - & 0.36 \\
Hartree-Fock + GW , ref. [15] & 1.86 & - \\
\hline \hline
\end{tabular}

\section{FIGURES}

Figure 1. Quasi-particle band structure of trans-polyacetylene (a) and poly-phenylene-vinylene (b), obtained from a GW quasiparticle calculation. In both cases, the polymer is treated as an isolated chain.

Figure 2. Absorption strength of trans-polyacetylene, with polarization vector along the chain direction. The transverse absorption spectrum is 3 orders of magnitude weaker (see text). The dashed (solid) line is the spectrum neglecting (including) electron-hole interaction.

Figure 3. Band structure of crystalline trans-polyacetylene, obtained from DFT-GGA calculation.

Figure 4. Same as Figure 2 for poly-phenylene-vinylene (PPV).

Figure 5. Real-space exciton wavefunction, showing the distribution of the electron relative to the hole. The $x$ axis is distance between electron and hole (the distribution is averaged over a plane perpendicular to the chain, and the hole is positioned at $\mathbf{r}_{h}=0$ ). Upper panel shows a bound state and lower panel shows a resonant state. Both are optically active.

[1] A. J. Heeger, S. Kivelson, J. R. Schrieffer, and W. P. Su, Rev. Mod. Phys. 60, 781 (1988)

[2] M. S. Hybertsen and S. G. Louie, Phys. Rev. Let. 55, 1418 (1985); Phys. Rev. B 34, 5390 (1986)

[3] S. G. Louie, "Ab-initio calculation of Electron Excitation Energies in Solids" in Modelling and Simulation for Materials Design, eds. S. Nishijima and H. Onodera (NRIM, Japan, 1996) p. 61 and reference therein

[4] M. Rohlfing and S. G. Louie, Phys. Rev. Lett. 80, 3320 (1998); Phys. Rev. Lett. 81, 2312 (1998); Phys. Rev. Lett. 83, 856 (1999) and reference therein

[5] L. X. Benedict, E. L. Shirley and R. B. Bohn, Phys. Rev. Lett. 80, 4514 (1998) ; Phys. Rev. B 59, 5441 (1999)

[6] S. Albrecht, L. Reining, R. Del Sole and G. Onida, Phys. Rev. Lett. 80, 4510 (1998)

[7] G. Strinati, Phys. Rev. B29, 5718 (1984)

[8] M. Rohlfing and S. G. Louie, Phys. Rev. Lett. 82, 1959 (1999)

[9] P. Vogl and D. K. Campbell, Phys. Rev. Lett. 62, 2012 (1989) ; Phys. Rev. B. 41, 12797 (1990)

[10] M. Rohlfing, P. Krüger and J. Pollmann, Phys. Rev. Lett. 75, 3489 (1995); Phys. Rev. B 52, 1905 (1995)

[11] L. Hedin, Int. J. Quantum Chem. 56, 445 (1995)

[12] C. S. Yannoni and T. C. Clarke, Phys. Rev. Lett. 51, 1191 (1983)

[13] Q. Zhu et al., Solid State Commun. 83, 179 (1992)

[14] G. Leising, Phys. Rev. B 38, 10313 (1988)

[15] E. C. Ethridge et al., Phys. Rev. B 53, 3662 (1996)

[16] D. A. Halliday et al. Synth. Met. 55-57, 954 (1993)

[17] S. Mukamel et al., Science 277, 781 (1997)

[18] Yu. N. Gartstein, M. J. Rice and E. M. Conwell, Phys. Rev. B 52, 1683 (1995); M. Chandross et a., Phys. Rev B 55, 1486 (1997)

[19] M. Chandross and S. Mazumdar, Phys. Rev B 55, 1497 (1997); N. Kirova, S. Brazovskii and A. R. Bishop, Synth. Met. 100, 29 (1999) 

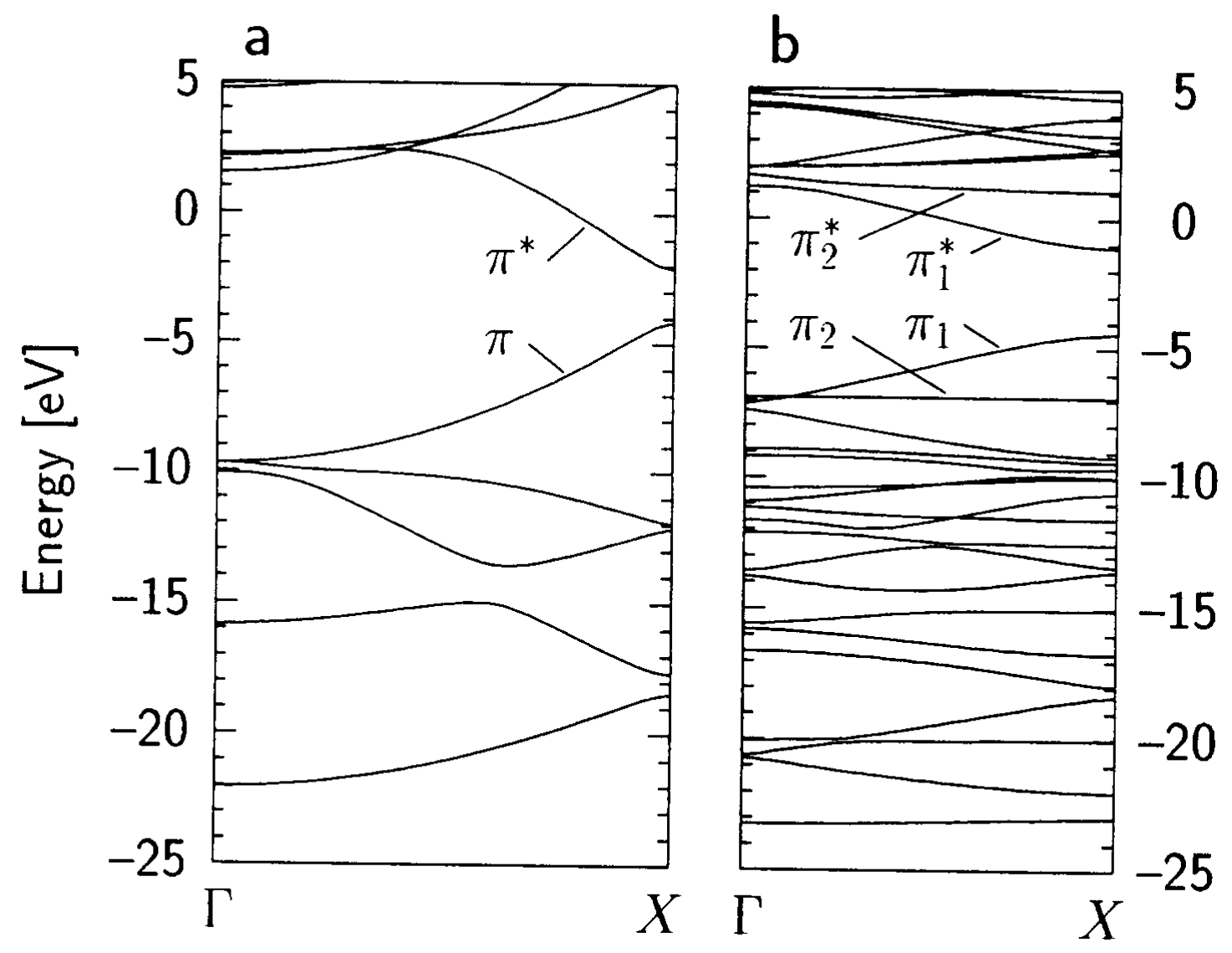

FIG. 1.

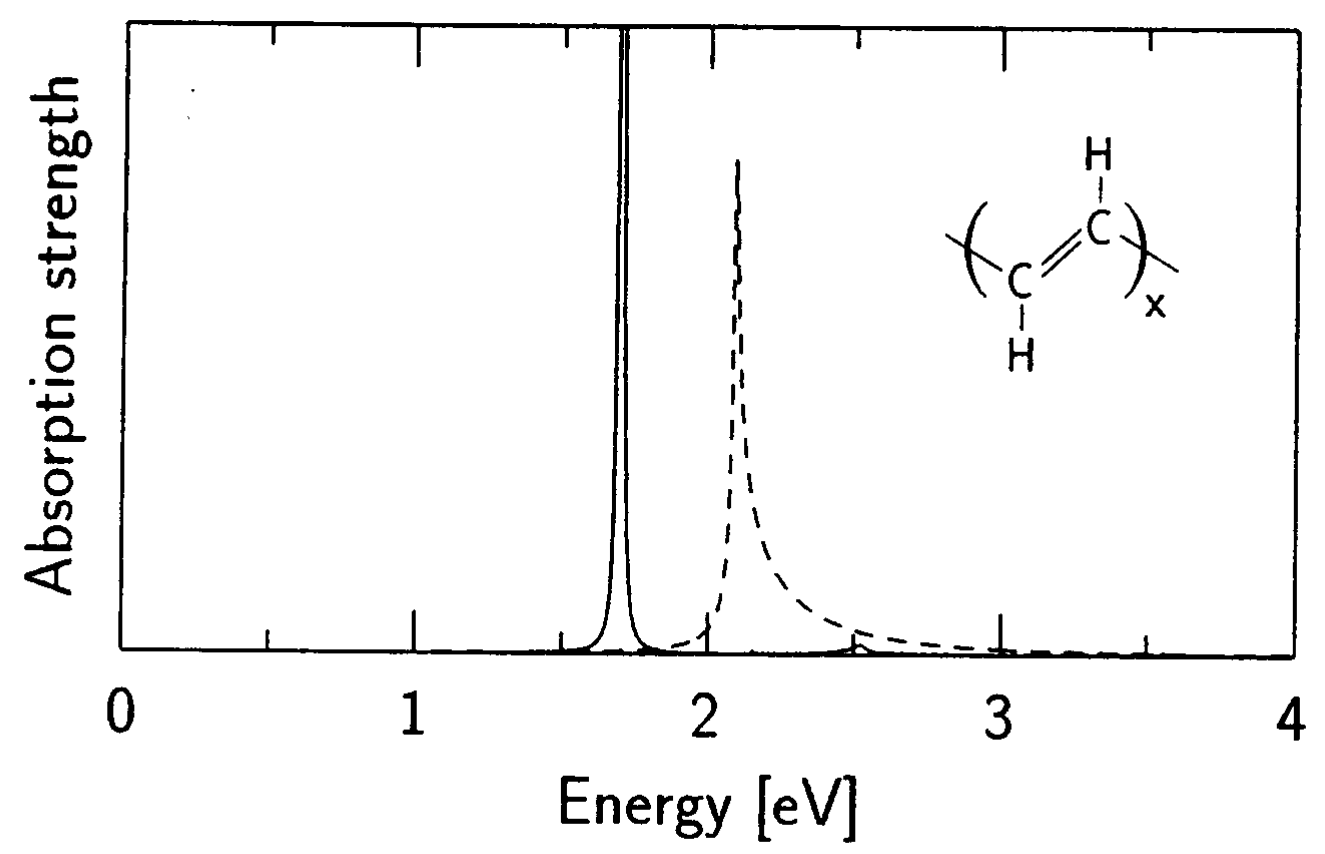

FIG. 2. 

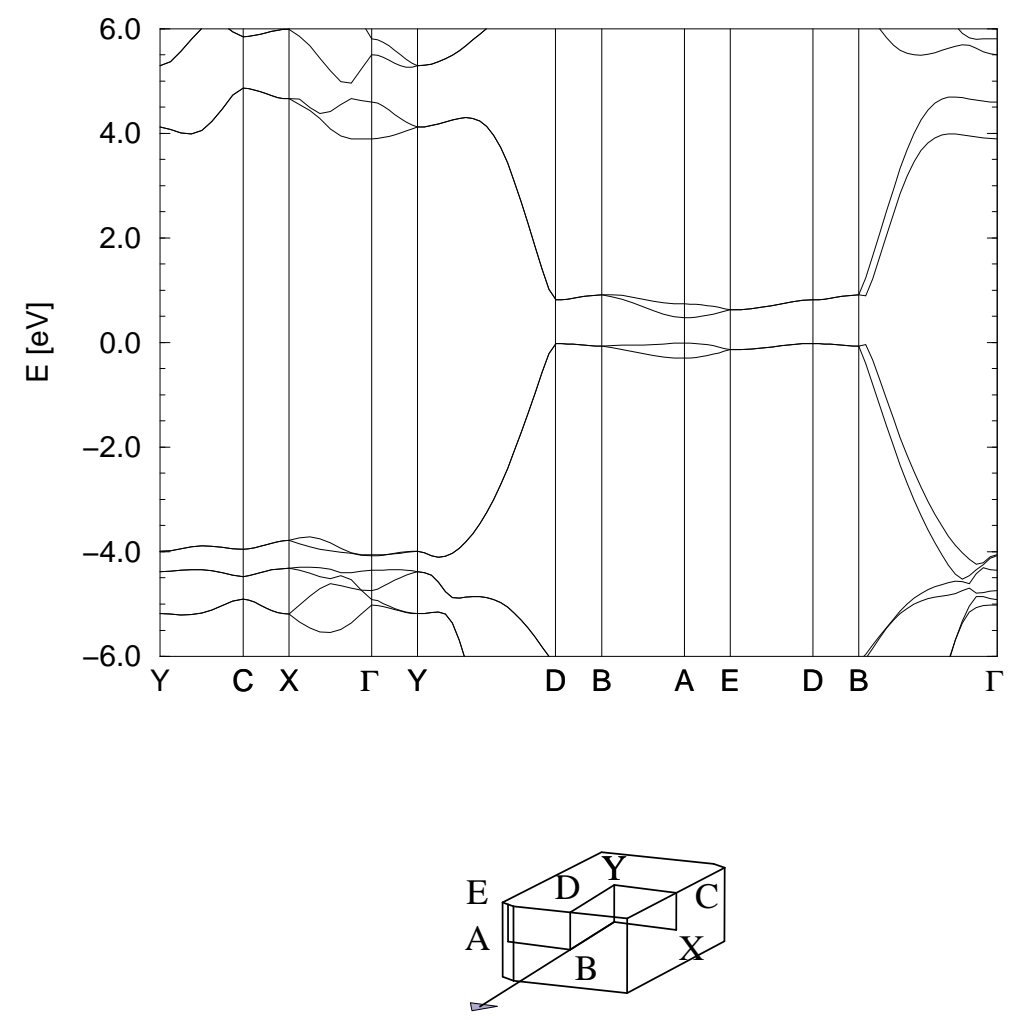

FIG. 3. 


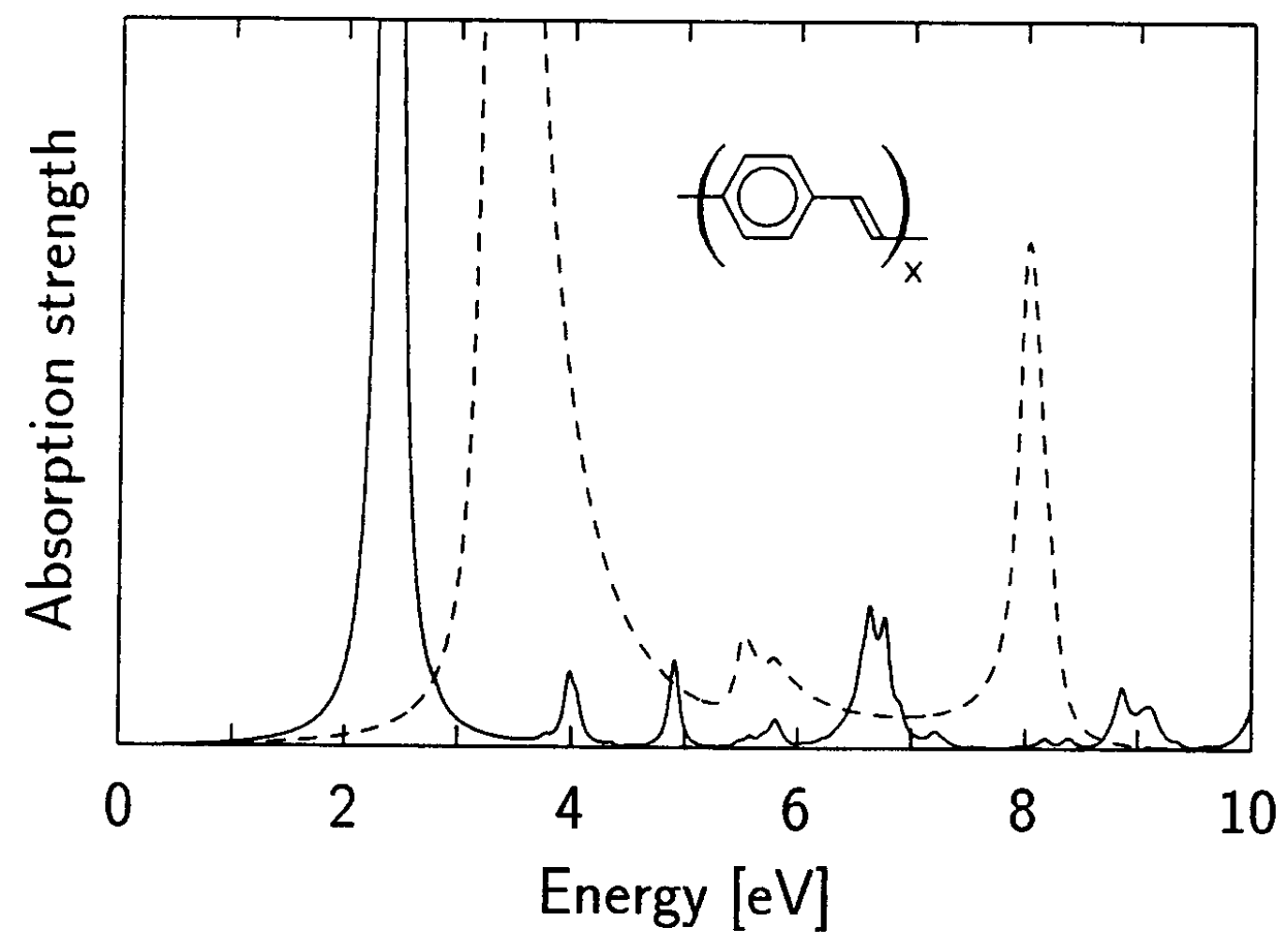

FIG. 4 


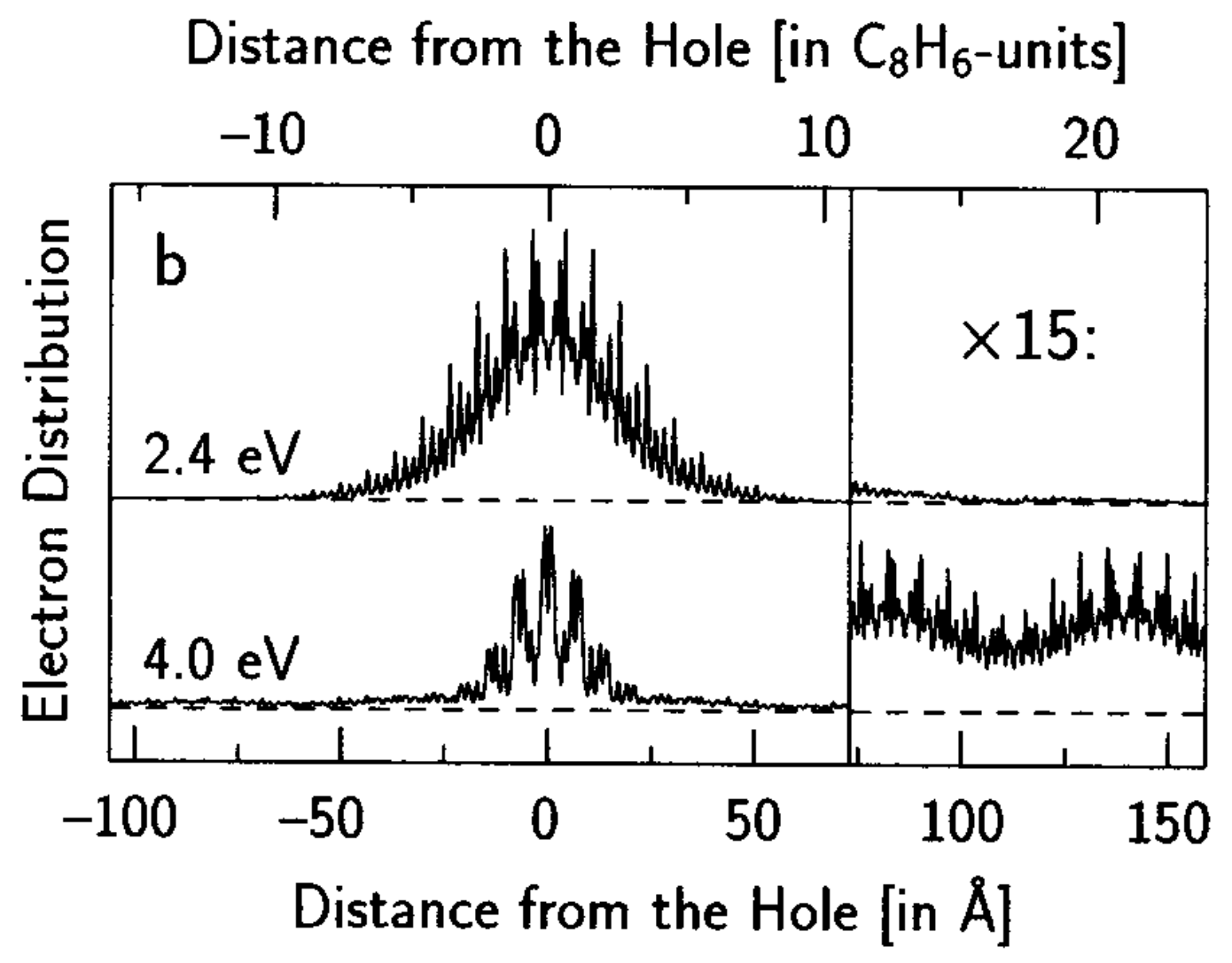

FIG. 5. 